\title{
Derivational Error of Albert Einstein
}

\author{
B. Ravi Sankar, \\ Scientist, MPAD/MDG, ISRO Satellite Centre, Bangalore-560017, INDIA
}

\begin{abstract}
The purpose of this paper is to point out a major derivational error in Albert Einstein's 1905 paper titled "ON THE ELECTRODYNAMICS OF MOVING BODIES". An alternate expression for coordinate transformation is derived which shows that the time co-ordinate of the moving clock cannot be expressed in terms of the temporal and spatial co-ordinate of the stationary system.

Keywords - Special theory of relativity, on the electrodynamics of moving bodies, coordinate transformation, kinematical part, definition of simultaneity.
\end{abstract}

\section{INTRODUCTION}

Albert Einstein published three papers in the year 1905. Among them the paper on photo electric effect yielded him the Nobel Prize. He was not awarded Nobel Prize for his celebrated general theory of relativity or special theory of relativity. The 1905 paper titled "ON THE ELECTRODYNAMICS OF MOVING BODIES" is the source of special theory of relativity. For a quiet long time, people have speculated that there is something wrong in special theory of relativity. The purpose of this paper is not to disprove or dispute the special theory relativity but to point out a major derivational error in the section titled "Theory of the Transformation of Coordinates and Times from a Stationary System to another System in Uniform Motion of Translation relatively to the Former" under the "KINEMATICAL PART' of the paper "ON THE ELECTRODYNAMICS OF THE MOVING BODIES” [1].

\section{MAJOR DERIVATIONAL ERROR}

The first assumption Einstein made in that section is $\frac{1}{2}\left(\tau_{0}+\tau_{2}\right)=\tau_{1}$. The aim of this paper is to prove that he has wrongly assumed. The aim is to prove $\frac{1}{2}\left(\tau_{0}+\tau_{2}\right) \neq \tau_{1}$ or equivalently $\left(\tau_{0}+\tau_{2}\right) \neq 2 \tau_{1}$. In order to prove this one need to thoroughly understand the figure 1 as well as the KINEMATICAL PART of the paper "ON THE ELECTRODYNAMICS OF MOVING BODIES'.

Let us in "Stationary" space take two system of co-ordinates, i.e. two systems, each of three rigid material lines, perpendicular to one another, and issuing from a point (origin $\mathrm{O}$ or o). Let the axes of $\mathbf{X}$ of the two systems coincide, and their axes of $\mathbf{Y}$ and $\mathbf{Z}$ be parallel. Let each system be provided with a rigid measuring-rod and a number of clocks, and let the two measuring-rods, and likewise all the clocks of the two systems, be in all respects alike [1].

Now to the origin (o) of one of the two systems $(k)$ let a constant velocity $v$ be imparted in the direction of the increasing $x$ of the other stationary system $(\mathbf{K})$, and let this velocity be communicated to the axes of the co-ordinates, the relevant measuring -rod, and the clocks. To any time of stationary system $\mathbf{K}$ there then will correspond a definite position of the axes of the moving system, and from reasons of symmetry we are entitled to assume that the motion of $k$ may be such that the axes of the moving system are at the time $t$ (this " $t$ ' always denotes a time of the stationary system) parallel to the axes of the stationary system [1-page5].

To any system of values $x, y, z, t$, which completely defines the place and time of an event in the stationary system, there belongs a system of values $\xi, \eta, \zeta, \tau$, determining that event relatively to the system $\mathrm{k}$, and our task is to find the system of equations connecting these quantities [1]. The foregoing discussion is pictorially represented in figure 1. 


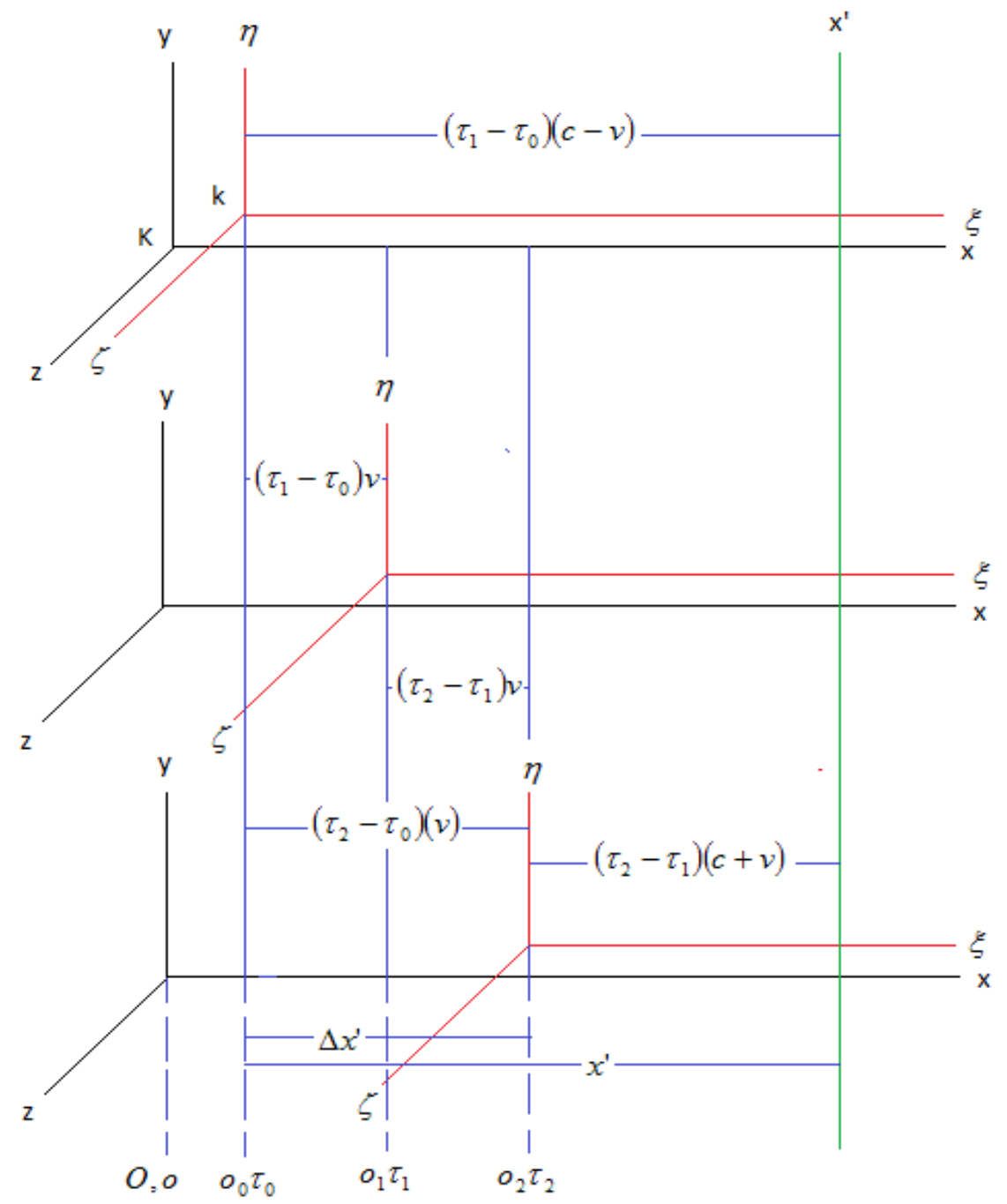

Figure 1: Pictorial representation of stationary and moving co-ordinate system.

From the origin of the moving system $k$ let a ray be emitted at the time $\tau_{0}$ along the $\mathbf{X}$-axis to $x^{\prime}$ and at the time $\tau_{1}$ be reflected thence to the origin of the co-ordinates, arriving at the time $\tau_{2}$ [1-page6]; we must prove that $\frac{1}{2}\left(\tau_{0}+\tau_{2}\right) \neq \tau_{1}$. Before proceeding further, the nomenclature of the figure is discussed.

- Black color co-ordinate represents the stationary system.

- Red color co-ordinate represents the moving system.

- Green line represents the point at which ray is reflected.

- $O, o$ Represents origin of stationary and moving system at initial condition.

- $o_{0} \tau_{0}$ Represents the origin of moving system when the ray is emitted w.r.to stationary system

- $o_{1} \tau_{1}$ Represents the origin of moving system when the ray is received at $x^{\prime}$

- $\mathrm{O}_{2} \tau_{2}$ Represents the origin of moving system when the ray is received back at the origin of moving system.

- $\mathrm{XYZ}$ represents the stationary co-ordinate system.

- $\xi \eta \zeta$ Represents the moving co-ordinate system.

- $\tau$ Represents the co-ordinate time of the uniformly moving system along X-direction.

Now the stage is set to point out the derivational error. Reminder: the error which we want to point out is $\frac{1}{2}\left(\tau_{0}+\tau_{2}\right) \neq \tau_{1}$. Before proceeding to the derivational aspects, the following points are worth mentioning. 
- The ongoing ray travels a distance of $x^{\prime}$ with a velocity of $(c-v)$

- By the time the reflected ray reaches the origin, the origin has shifted a distance of $\Delta x^{\prime}$ and hence the reflected ray travels a distance lesser than $x^{\prime}$ by an amount $\Delta x^{\prime}=\left(\tau_{2}-\tau_{0}\right) v$. This point has not been noticed by Albert Einstein.

- $\tau_{0}$ is the reading of the clock at the time of emission.

- $\tau_{1}$ is the reading of the clock at the time of reflection at $x^{\prime}$.

- $\tau_{2}$ is the reading of the clock at the time of reception at the origin.

- Always $\tau$ represents the time in the moving clock and $t$ represents the time in the stationary clock.

Referring to figure 1 , the following equations are derived.

$$
\begin{aligned}
& \tau_{0}=\tau_{1}-\frac{x^{\prime}}{c-v} \\
& \tau_{2}=\tau_{1}+\frac{x^{\prime}-\Delta x^{\prime}}{c+v} \\
& \Delta x^{\prime}=\left(\tau_{2}-\tau_{0}\right) v \\
& x^{\prime}=\left(\tau_{1}-\tau_{0}\right)(c-v)
\end{aligned}
$$

Upon adding equation (1) and equation (2), one gets the following equation.

where

$$
\tau_{0}+\tau_{2}=2 \tau_{1}+\frac{x^{\prime}-\Delta x^{\prime}}{c+v}-\frac{x^{\prime}}{c-v}=2 \tau_{1}+A(\tau, c, v)
$$

$$
A(\tau, c, v)=\frac{x^{\prime}-\Delta x^{\prime}}{c+v}-\frac{x^{\prime}}{c-v}
$$

Dividing equation (5) by 2 , we get the following equation.

$$
\frac{1}{2}\left(\tau_{0}+\tau_{2}\right)=\tau_{1}+\frac{x^{\prime}-\Delta x^{\prime}}{2(c+v)}-\frac{x^{\prime}}{2(c-v)}
$$

From equation (7), it is clear that $\frac{1}{2}\left(\tau_{0}+\tau_{2}\right) \neq \tau_{1}$. It is also clear that $\left(\tau_{0}+\tau_{2}\right)=2 \tau_{1}$ only when $v=0$.

So it is clear form equation (7) that Einstein made a wrong assumption [1-page 6] at the very beginning of his derivation. With this, this section is concluded. An alternate derivation for the coordinate transformation follows in the following sections. Readers are requested to thoroughly understand this section before proceeding further.

\section{DERIVATION OF $A(\tau, c, v)$}

The additional term appearing along with $\tau_{1}$ in equation (5) is derived in this section. Substituting equation (3) \& (4) in equation (6), one gets the following expression for $A(\tau, c, v)$.

$$
\begin{gathered}
A(\tau, c, v)=\frac{x^{\prime}}{c+v}-\frac{x^{\prime}}{c-v}-\frac{\Delta x^{\prime}}{c+v} \\
A(\tau, c, v)=\frac{\left(\tau_{1}-\tau_{0}\right)(c-v)}{c+v}-\frac{\left(\tau_{1}-\tau_{0}\right)(c-v)}{c-v}-\frac{\left(\tau_{2}-\tau_{0}\right) v}{c+v}
\end{gathered}
$$

Upon simplifying the above equation, we get the following expression for $A(\tau, c, v)$.

$$
A(\tau, c, v)=\frac{3 v \tau_{0}-2 v \tau_{1}-v \tau_{2}}{c+v}
$$

Substituting equation (10) back in equation (5), we get the following expression. 


$$
\tau_{0}+\tau_{2}=2 \tau_{1}\left(\frac{c}{c+v}\right)+\left(\frac{v\left(3 \tau_{0}-\tau_{2}\right)}{c+v}\right) \neq 2 \tau_{1}
$$

From the above equation it is clear that $\left(\tau_{0}+\tau_{2}\right) \neq 2 \tau_{1}$. It is also clear that $\left(\tau_{0}+\tau_{2}\right)=2 \tau_{1}$ only when $v=0$.

\section{An Alternate Derivation Of The CO-Ordinate Transformation}

Upon simplifying equation (11), we get the following expression

$$
(c-2 v) \tau_{0}+(c+2 v) \tau_{2}=2 c \tau_{1}
$$

Before proceeding further, the following points are worth mentioning.

- $\quad$ The argument of $\tau_{0}$ are $\tau(0,0,0, t)$

- The argument of $\tau_{1}$ are $\tau\left(x^{\prime}, 0,0, t+\frac{x^{\prime}}{c-v}\right)$

- The argument of $\tau_{2}$ are $\tau\left(\Delta x^{\prime}, 0,0, t+\frac{x^{\prime}}{c-v}+\frac{x^{\prime}-\Delta x^{\prime}}{c+v}\right)$, Albert Einstein has overlooked the argument of $\tau_{2}$ i.e. he did not notice that the origin of the moving co-ordinate has shifted a distance of $\Delta x^{\prime}$ during the rays flight time forth and back. He also overlooked the time $t$ argument of $\tau_{2}$, where he has substituted $t+\frac{x^{\prime}}{c-v}+\frac{x^{\prime}}{c+v}$ instead of $t+\frac{x^{\prime}}{c-v}+\frac{x^{\prime}-\Delta x^{\prime}}{c+v}$. The factor $\Delta x^{\prime}$ should appear in the time coordinate because the reflected ray travels $\Delta x^{\prime}$ lesser distance than the emitted ray.

Upon substituting the arguments of $\tau$ in equation (12), one gets the following expression [1-page6].

$$
(c-2 v) \tau(0,0,0, t)+(c+2 v) \tau\left(\Delta x^{\prime}, 0,0, t+\frac{x^{\prime}}{c-v}+\frac{x^{\prime}-\Delta x^{\prime}}{c+v}\right)=2 c \tau\left(x^{\prime}, 0,0, t+\frac{x^{\prime}}{c-v}\right)
$$

Before proceeding further, $\Delta x^{\prime}$ should be expressed in terms of $x^{\prime}$. That is done in the following steps. Subtracting equation (1) from equation (2), the following equation is obtained.

$$
\begin{aligned}
& \tau_{2}-\tau_{0}=\frac{x^{\prime}}{c+v}+\frac{x^{\prime}}{c-v}-\frac{\Delta x^{\prime}}{c+v} \\
& \left(\tau_{2}-\tau_{0}\right)+\frac{\Delta x^{\prime}}{c+v}=\frac{x^{\prime}}{c+v}+\frac{x^{\prime}}{c-v}
\end{aligned}
$$

Substituting for $\Delta x^{\prime}$ from equation (3), the above equation simplifies as follows.

$$
\begin{aligned}
& \left(\tau_{2}-\tau_{0}\right)\left(1+\frac{v}{c+v}\right)=\frac{2 c x^{\prime}}{(c+v)(c-v)} \\
& \left(\tau_{2}-\tau_{0}\right)=\frac{2 c x^{\prime}}{(c+2 v)(c-v)}
\end{aligned}
$$

Substituting equation (17) in equation (3), the expression for $\Delta x^{\prime}$ is obtained as follows.

$$
\Delta x^{\prime}=\left(\tau_{2}-\tau_{0}\right) v=\frac{2 c v x^{\prime}}{(c+2 v)(c-v)}=\alpha x^{\prime}
$$

Where $\alpha$ is given by the following equation.

$$
\alpha=\frac{2 c v}{(c+2 v)(c-v)} .
$$


The time argument of $\tau_{2}$ also contains $\Delta x^{\prime}$ and hence it should be expressed in terms of $x^{\prime}$. That is done in the following steps. The time argument of $\tau_{2}$ is $t+\frac{x^{\prime}}{c-v}+\frac{x^{\prime}-\Delta x^{\prime}}{c+v}$ from equation (13). This $\Delta x^{\prime}$ should be eliminated before proceeding further. Let $\beta x^{\prime}=\frac{x^{\prime}}{c-v}+\frac{x^{\prime}-\Delta x^{\prime}}{c+v}$. Upon substituting $\Delta x^{\prime}$ from equation (18) in $\beta$, the expression for $\beta$ simplifies as follows.

$$
\beta=\frac{1}{c-v}+\frac{1}{c+v}-\frac{\alpha}{c+v}
$$

Upon substituting $\alpha$ from equation (19), the above expression reduces as follows.

$$
\beta=\frac{2 c}{(c-v)(c+2 v)}
$$

Now the stage is set for deriving the equation of co-ordinate transformation. Equation (13) reduces as follows.

$$
(c-2 v) \tau(0,0,0, t)+(c+2 v) \tau\left(\alpha x^{\prime}, 0,0, t+\frac{x^{\prime}}{c-v}+\frac{x^{\prime}}{c+v}-\frac{\alpha x^{\prime}}{c+v}\right)=2 c \tau\left(x^{\prime}, 0,0, t+\frac{x^{\prime}}{c-v}\right)
$$

Further simplifying results in the following equation.

$$
(c-2 v) \tau(0,0,0, t))+(c+2 v) \tau\left(\alpha x^{\prime}, 0,0, t+\beta x^{\prime}\right)=2 c \tau\left(x^{\prime}, 0,0, t+\frac{x^{\prime}}{c-v}\right)
$$

Hence if $x^{\prime}$ is chosen infinitesimally small (As claimed by Einstein [1-page 6]), the above equation reduces as follows (upon expanding by Taylor series to the first order).

$$
(c+2 v)\left(\alpha \frac{\partial \tau}{\partial x^{\prime}}+\beta \frac{\partial \tau}{\partial t}\right)=2 c\left(\frac{\partial \tau}{\partial x^{\prime}}+\frac{1}{c-v} \frac{\partial \tau}{\partial t}\right)
$$

Since $(c+2 v) \beta=\frac{2 c}{c-v}$, the above equation reduces as follows.

$$
\begin{aligned}
& (c+2 v) \alpha \frac{\partial \tau}{\partial x^{\prime}}-2 c \frac{\partial \tau}{\partial x^{\prime}}=0 \\
& \frac{\partial \tau}{\partial x^{\prime}}=0
\end{aligned}
$$

The solution of the above equation is $\tau=$ const. Hence Time cannot be co-ordinate transferred as claimed by Einstein. There exists a peculiar solution for equation (24).

The above equation reduces as follows.

$$
(c-2 v) \alpha \frac{\partial \tau}{\partial x^{\prime}}=2 c \frac{\partial \tau}{\partial x^{\prime}}
$$

$$
(c+2 v) \alpha=2 c
$$

Upon substituting $\alpha$ in the above equation one gets $v=c / 2$.

\section{CONCLUSION}

The theory of relativity (both special and general theory of relativity) is proven beyond doubt. The purpose of the manuscript is not to dispute special theory relativity. The objective of this paper is to prove that the mathematical method employed by Albert Einstein to arrive at his equation is wrong and hence it is fulfilled.

\section{REFERENCES}

[1] Albert Einstein, On the electrodynamics of moving bodies, (Zur Electrodynamik bewegter Körper) Annalen der Physik, 1905, http://www.fourmilab.ch/einstein/specrel/specrel.pdf page 5-6. 